\title{
Feasibility study of the microforming combined with selective chemical vapor deposition
}

\author{
Kazushi Koshimizu*, Tetsuhide Shimizu, and Ming Yang \\ Tokyo Metropolitan University, 6-6 Asahigaoka, Hino-shi, Tokyo 191-0065, Japan
}

Received 7 October 2015 / Accepted 10 October 2015

\begin{abstract}
Microforming has been received much attention in the recent decades due to the wide use of microparts in electronics and medical applications. For the further functionalization of these micro devices, high functional surfaces with noble metals and nanomaterials are strongly required in bio- and medical fields, such as bio-sensors. To realize an efficient manufacturing process, which can deform the submillimeter scale bulk structure and can construct the micro to nanometer scale structures in one process, the present study proposes a combined process of microforming for metal foils with a selective chemical vapor deposition (SCVD) on the active surfaces of the working material. To demonstrate feasibility of this proposed process, feasibility of SCVD of functional materials onto active surfaces of titanium (Ti) was investigated. CVD of iron (Fe) and carbon nanotubes (CNTs) which construct CNTs on the patterned surfaces of the active $\mathrm{Ti}$ and non-active $\mathrm{Ti}$ oxidation layers were conducted. Ti thin films on silicon substrate and Fe were used as working materials and functional materials respectively. CNTs were grown only on the Ti surface. Consequently, selectivity of the active surface of Ti to the synthesis of Fe particles in CVD was confirmed.
\end{abstract}

Key words: Microforming, Active surface, Selective chemical vapor deposition

\section{Introduction}

Due to attractive characteristics of microforming such as low cost, high productivity and stability of the process, demand for micro press-forming is increasing for realizing manufacture of small devices made by metallic parts, taking advantage of good properties of metals, such as mechanical strengths, electrical conductivity and chemical resistance. Especially for medical devices, biocompatibility of metal has been highly required, and its miniaturization technology is needed. Additionally bio-devices with noble metal or nanomaterials, such as gold $(\mathrm{Au})$, platinum $(\mathrm{Pt})$ and Carbon nanotubes (CNTs) on the surface, are often used in bio-technology by exerting good functions like detecting antibody [1] or catalytic reaction [2]. And, the nano-structuration technologies for functional surface are also required to increase these functions [3]. Therefore, the process for the functional surface is importance in the fabrication of bio- and medical devices.

In the present study, a process with the combination of micro press forming and fabrication of the functional surface for metal foils was proposed. This process can realize the submillimeter structure of metal foils and micro to nanometer

*e-mail: koshimizu-kazushi@ed.tmu.ac.jp structures of functional materials in one step. In this process, selective chemical vapor deposition (SCVD) for active surface was selected to fabricate the functional surfaces. Mohammadi et al. reported that, active surface of $\mathrm{Si}$ has better selectivity than non-active surface of $\mathrm{SiO}_{2}$ in metal CVD process. Thus, these metal thin films can be deposited selectivity for oxide and non-oxide patterned surface [4]. As shown in Figure 1, a deformation process may break down the oxidation layer of the metal foil. Thus, active and non-active surfaces may also be patterned on the metal foil, and the functional materials can be deposited only on the cracking area by using SCVD.

The aim of this study is to demonstrate the feasibility of the combined process. Thus, this work entailed three tasks. The first task was to develop the system for a combing process of micro forming of metal foils with SCVD. The second task was to investigate the behavior of straining and effect of the thickness of oxidation layer on the cracking by the bulging test on the metal foil. In this study, Ti foils were used as the working material. The last task was to investigate the feasibility of SCVD on the active and non-active surfaces by depositing functional materials on these surfaces. In this third task, active and non-active surfaces were prepared by a partial oxidation process and an oxidation-reduction process. Furthermore, possibility of selective deposition of the functional materials was 


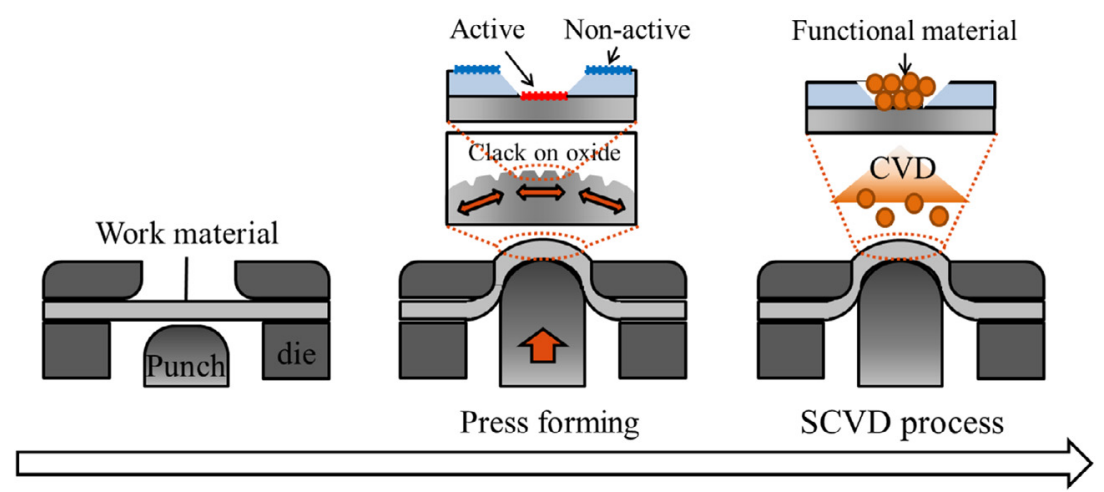

Figure 1. Schematic illustration of the proposed process of the press forming combined with selective chemical vapour deposition.

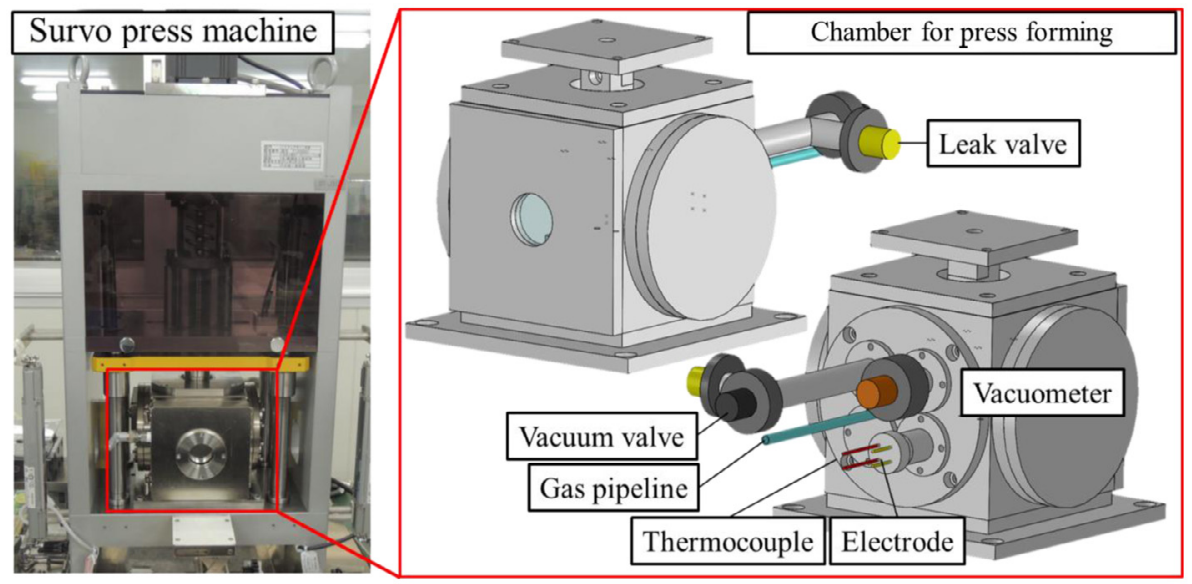

Figure 2. The appearance and schematic illustration of developed press forming system.

assessed by a synthesizing process of carbon nanotubes (CNTs) using the catalyst effect of the functional materials.

\section{System development for press forming combined with CVD}

To conduct CVD process with micro press forming, this study needs new press forming system which can realize control of the atmosphere, carrying gas or precursor of CVD, heat the work materials, control the temperature of the work material and micro deforming system. Thus, new system for press forming combined with CVD was developed.

As shown in Figure 2, the system is mainly consisted of a servo press machine and a small stainless steel chamber. This servo press machine (SEKI Corporation) has a punch stroke with high resolution of $0.001 \mathrm{~mm} / \mathrm{s}$ to adjust for micro forming. This chamber was designed in a small size (height is $200 \mathrm{~mm}$, width is $140 \mathrm{~mm}$ ) to be set in limited area of the press machine. Additionally, vacuometer, vacuum valve, gas pipeline, and leak valve were equipped in the back side of chamber for controlling atmosphere and carrying gas or precursor of CVD. The system with the chamber could realize the inert gas atmosphere of argon (Ar) gas to protect the working material from being exposed to the air and to carrying the precursors with maximum $500 \mathrm{ccm}$.
As shown in Figure 3, designed bulging die was placed inside the chamber. In this present study, bulge forming was adopted because of the easiness for deforming the surface of the working material. The loadcell was embedded into the forming die in order to obtain the force signal and the information was further used for an analysis aiming at controlling straining of the working material. Additionally, resistance heating was realized by replacing the holding part with an electrode. This system could heat the die with a maximum power of $800 \mathrm{~W}$. The temperatures were measured with thermocouples in order to implement heating control.

Thus, this developed system could realize bulge forming and CVD on the working material at the same time and could control the parameters for CVD to realize the proposed process and suitable conditions.

\section{Experiment}

\subsection{Verification of the cracks by plastic deformation}

To confirm influence from the thickness of the oxidation layer on the crack-forming process, different thicknesses of the oxidation layer on the Ti foil were prepared. Thickness of a Ti foil was $0.1 \mathrm{~mm}$. Ti foils were annealed at $700{ }^{\circ} \mathrm{C}$ 

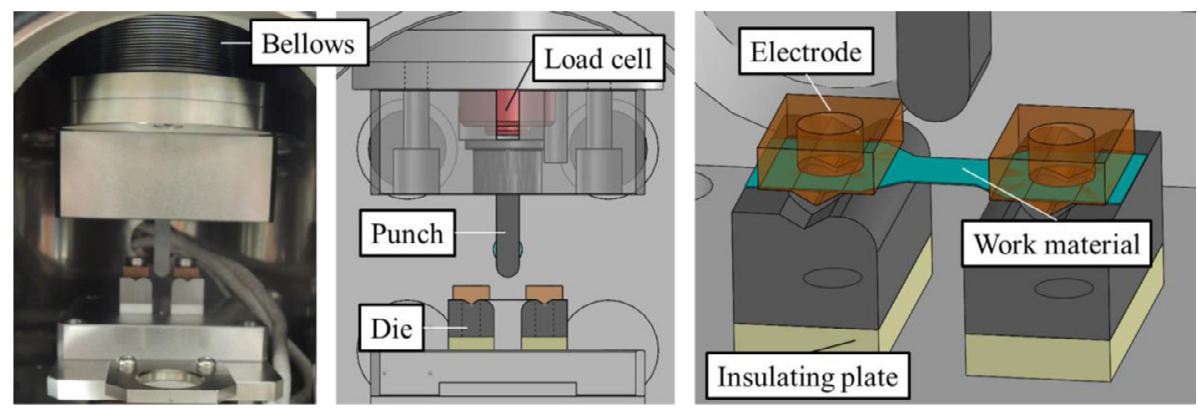

Figure 3. The appearance and schematic illustration of dies for bulging process.

Table 1. Conditions of bulge forming.

\begin{tabular}{lc}
\hline Punch stroke $[\mathrm{mm}]$ & 2 \\
Strain rate $[\mathrm{mm} / \mathrm{s}]$ & 0.01 \\
Thickness of oxidation layer $[\mathrm{nm}]$ & 20 (as received), 100, 150 \\
\hline
\end{tabular}

for 180 and $300 \mathrm{~s}$ respectively to grow the oxidation layer to a thickness of 100 and $150 \mathrm{~nm}$ respectively. After the annealing, these $\mathrm{Ti}$ foils were deformed by the bulge forming system. Those conditions were shown in Table 1.

\subsection{Feasibility of SCVD on the active and non-active surfaces}

To investigate the selectivity of the CVD process for active and non-active surfaces, CVD was conducted to pattern the Ti and $\mathrm{TiO}_{2}$ surfaces in the CVD furnace with a quarts tube. As shown in Figure 4, $\mathrm{TiO}_{2}$ film was patterned on a $\mathrm{Ti}$ foil by using an oxide plasma machine with a cover plate. After preparing this patterned substrate, oxidation-hydrogen reduction process was conducted to remove thin oxidation films on the Ti surface. Thus, active surfaces of Ti were exposed. In this study, Iron nanoparticles were used as functional materials in the CVD process. Fe CVD process was conducted with precursor of ferrocene $\left[\mathrm{Fe}\left(\mathrm{C}_{5} \mathrm{H}_{5}\right)_{2}\right]$. Since the Fe particles will work as a catalyst for growth of CNTs, CNTs were synthesized to confirm the deposited location of the Fe nanoparticles on the substrate.

\section{Results and discussion}

\subsection{Verification of the cracks by plastic deformation}

The surfaces of Ti foils after deforming were observed by a scanning electron microscope (SEM). As shown in Figures 5b and $5 \mathrm{c}$, there were some cracks on the surface of the Ti foils. It was considered that these cracks were caused by the difference of the elastic modulus of the bulk material and the oxidation layer of Ti. Additionally, the widths of the cracks (shown in Figure 5c) became larger than that shown in Figure 5b. It indicates that the behaviour of the cracks resulted from the increase of the strain and thickness of the oxidation layer. Therefore, influence of the strain in the substrate material and thickness of the oxidation layer for cracking can be confirmed. To confirm it, finite element analysis of bulge forming and calculation for cracking were conducted, which was effected using FE software ABAQUS.

The schematic illustration of the FE model is given in Figure 6a. Table 2 lists the mechanical properties of the Ti foil and FE modelling conditions. In the FE model, mesh size of the specimens was $25 \mu \mathrm{m} \times 25 \mu \mathrm{m}$ and the punch stroke $2 \mathrm{~mm}$ (from the punch initial position to the contact with the Ti foil by the punch). As shown in Figure $6 \mathrm{~b}$, the high strain occurred around the corner radius of the punch.

Latella et al. [5] reported that the crack width of the oxidation layer could be observed at the area where the higher straining occurred. A cracking area was considered to be exposed as an active surface of the work materials. The crack width of the oxidation layer can be estimated by the following equation (1) [5],

$$
C_{s}=\frac{\sigma_{c} t}{\tau},
$$

where $C_{s}$ is the crack width, $\sigma_{c}$ the fracture strength, $t$ thickness of the oxidation layer, and $\tau$ the interfacial shear strength. As shown in Figure 7, when thickness of the oxidation layer was $3 \mathrm{~nm}$ [6], $100 \mathrm{~nm}$ and $150 \mathrm{~nm}$, the crackwidth increased with the level of the strain and the thickness. From these results, the width of the crack was confirmed to be close to the result of calculation.

\subsection{Feasibility of SCVD process for active and non-active surface}

As shown in Figure 8, after the CNTs CVD, the boundary line between the $\mathrm{Ti}$ surfaces and the $\mathrm{TiO}_{2}$ surface were observed by SEM and energy dispersive X-ray analyser (EDX). When the temperature in the CVD process was $750{ }^{\circ} \mathrm{C}$, the CNTs were synthesized uniformly on both surfaces. When this temperature was $450{ }^{\circ} \mathrm{C}$, the CNTs were synthesized only on the active surface of Ti. It was considered that the superiority of high surface energy of the activated Ti surface was kept at a lower deposition temperature, like $450{ }^{\circ} \mathrm{C}$. On the other hand, at a high deposition temperature, like $750{ }^{\circ} \mathrm{C}$, surface energy of the non-active surface of $\mathrm{TiO}_{2}$ was 

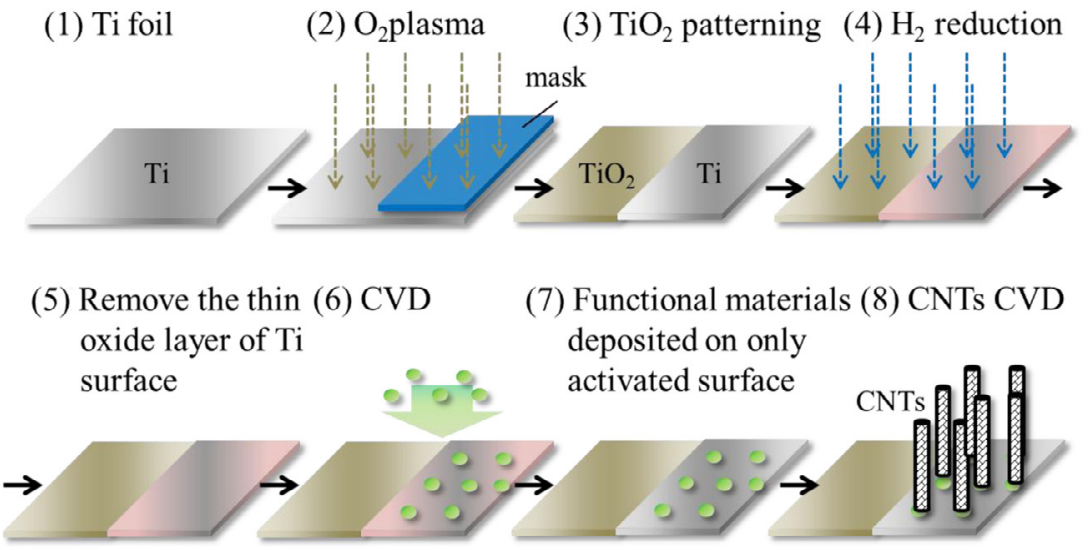

Figure 4. The schematic illustration of experiment to investigate the feasibility of SCVD.
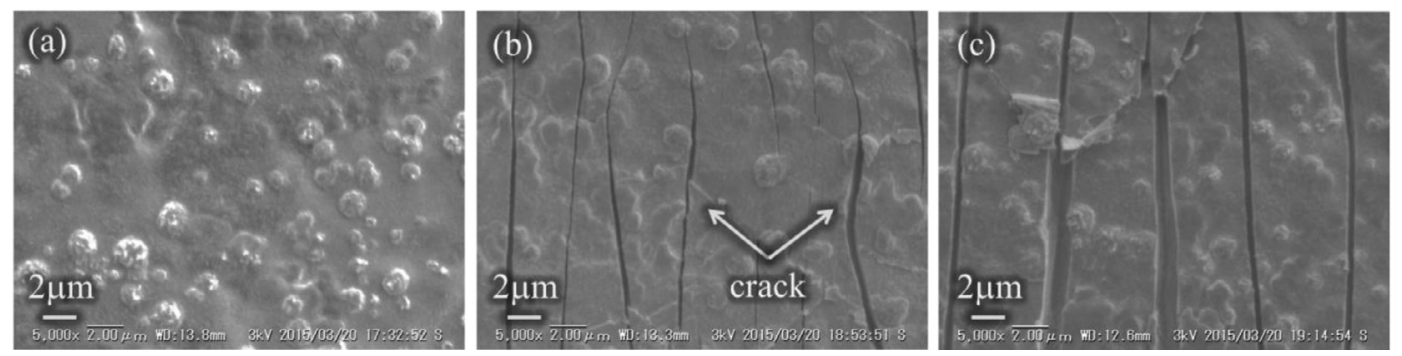

Figure 5. SEM images of deformed Ti foils (a) as received (thickness: $3 \mathrm{~nm}$ ), (b) deformed $6.2 \%$ of strain (thickness: $100 \mathrm{~nm}$ ), (c) deformed $6.2 \%$ of strain (thickness: $150 \mathrm{~nm}$ ).
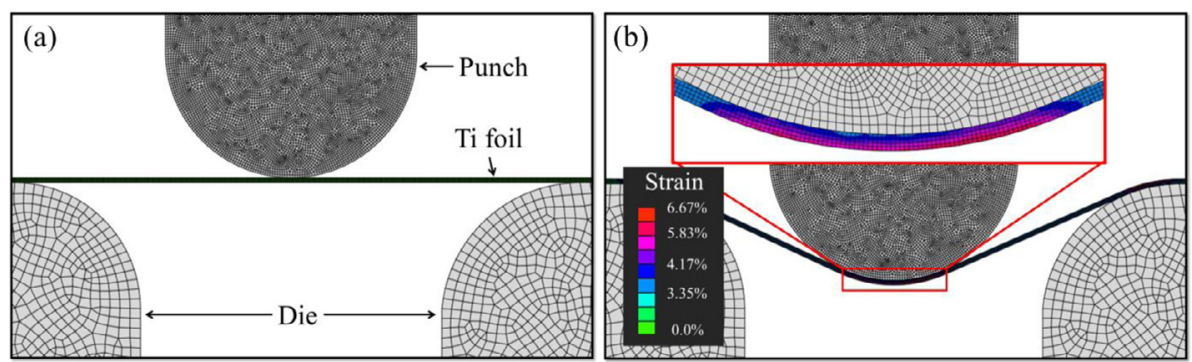

Figure 6. The schematic illustration of FEM (a) model, (b) distribution of strain under $2 \mathrm{~mm}$ punch stroke.

Table 2. Input mechanical properties of specimens and FE simulation conditions.

\begin{tabular}{lc}
\hline Specimen & $\mathrm{Ti}$ \\
\hline Mass density $[\mathrm{mg} / \mu \mathrm{m}]$ & $4.5 \times 10^{-9}$ \\
Young's modulus $[\mathrm{GPa}]$ & 110 \\
Yield stress [MPa] & 330 \\
Poisson's ratio & 0.34 \\
Friction coefficient & 0.1 \\
\hline
\end{tabular}

enhanced by heating energy. Therefore this selectivity of the active surface decreased. It indicates that Fe nanoparticles were deposited on the active surface of $\mathrm{Ti}$ more than that on the non-active surface of $\mathrm{TiO}_{2}$ at a low deposition temperature. This result suggests the feasibility of SCVD for an active surface of Ti.

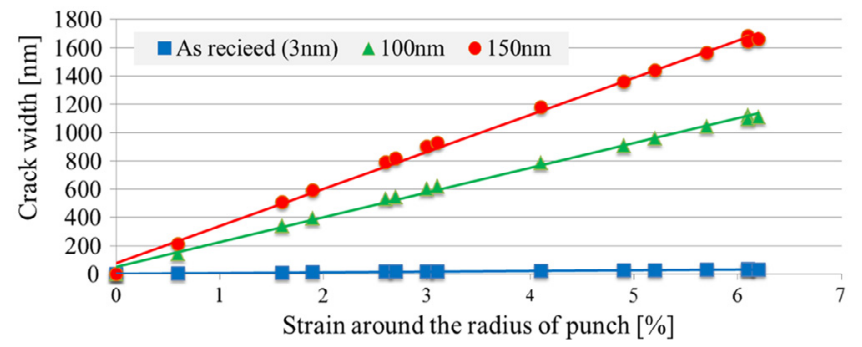

Figure 7. Calculation results of crack width of oxidation layer for different strain and thickness.

\subsection{Discussion}

From the results described in Sections 4.1 and 4.2, the relation of the oxide thickness and the level of the strain with the 


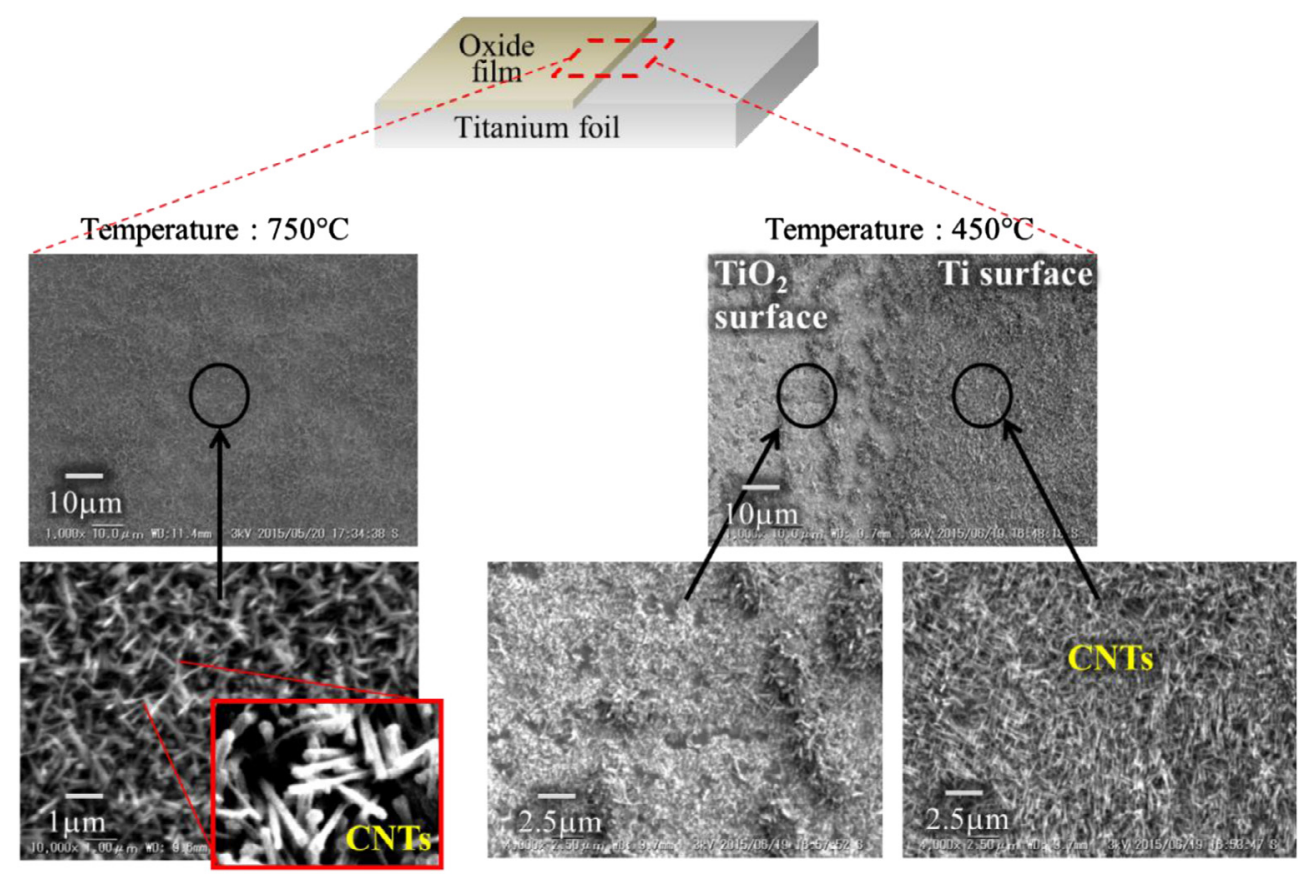

Figure 8. SEM image of $\mathrm{Ti}$ and $\mathrm{TiO}_{2}$ patterned surface after CNTs CVD process.

behaviour of cracking and feasibility of the SCVD process for active and non-active surfaces of Ti were confirmed. Because the surface under oxidation layer has higher surface energy than the surface of oxidation layer, the cracking area may contain higher active energy than that in the patterned surface with SCVD where there was no crack occurring. Thus, it is considered that the functional materials could be deposited more easily on the cracking area of the deformed oxidation layer.

It is expected that the developed system for implementing the combined process could realize favourable SCVD conditions such as temperature of $450{ }^{\circ} \mathrm{C}$ for the working material and other optimal conditions for the CVD process.

\section{Conclusions}

In this study, a combined process of micro bulging and selective chemical vapour deposition was proposed. To realize this process, a press forming system combined with CVD was developed. Press forming with controlled atmosphere and heating on the working material has been enabled. In addition, influences of straining and thickness of the oxidation layer of the Ti foil were investigated. Cracking of the oxidation layer was effected by the deformation of the Ti foil. It shows that the crack width increases with thickness of the oxidation layer. To investigate the feasibility of SCVD for active and nonactive surfaces of Ti, Fe CVD and CNTs CVD were conducted and CNTs were synthesized only on active surfaces of $\mathrm{Ti}$ at special CVD conditions. It shows that Fe nanoparticles were deposited only on the active surface when the Fe CVD process was carried out. Thus, the feasibility of Fe-SCVD on the active surface of Ti is confirmed.

In the following study, detail of the mechanism of cracking and factors influencing the control of formation of cracking patterns will be further investigated, and the process for creating patterns on actual micro parts will be tested.

\section{References}

1. X. Cao, et al., Gold nanoparticle-based signal amplification for biosensing, Anal. Biochem. 417 (2011) 1-16.

2. H. Zhao, et al., Pt catalyst supported on titanium suboxide for formic acid electrooxidation reaction, Int. J. Hydrogen Energy 39 (2014) 9621-9627.

3. X. Pang, et al., An amperometric glucose biosensor fabricated with Pt nanoparticle-decorated carbon nanotubes $/ \mathrm{TiO}_{2}$ nanotube arrays composite, Sens. Actuators B 137 (2009) 134-138.

4. V. Mohammadi, et al., Temperature dependency of the kinetics of PureB CVD deposition over patterned $\mathrm{Si} / \mathrm{SiO}_{2}$ surfaces, Microelectron. Eng. 125 (2014) 45-50.

5. B.A. Latella, et al., Fracture toughness and adhesion of thermally grown titanium oxide on medical grade pure titanium, Surf. Coat. Technol. 201 (2007) 6325-6331.

6. N.L. Do, et al., Determination of thicknesses of oxide films grown on titanium under argon irradiation by spectroscopic ellipsometry, J. Nucl. Mater. 447 (2014) 197-207.

Cite this article as: Koshimizu K, Shimizu T \& Yang M: Feasibility study of the microforming combined with selective chemical vapor deposition. Manufacturing Rev. 2015, 2, 24. 